\title{
COMBINING REMOTELY SENSED ENVIRONMENTAL CHARACTERISTICS WITH SOCIAL AND BEHAVIORAL CONDITIONS THAT AFFECT SURFACE WATER USE IN SPATIOTEMPORAL MODELLING OF SCHISTOSOMIASIS IN GHANA
}

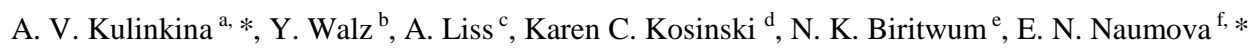 \\ a Dept. of Civil and Environmental Engineering, Tufts University, 200 College Ave, Medford, USA - alexandra.kulinkina@tufts.edu \\ ${ }^{\mathrm{b}}$ Institute for Environment and Human Security, United Nations University, Bonn, Germany - walz@ehs.unu.edu \\ ${ }^{\mathrm{c}}$ Dept. of Civil and Environmental Engineering, Tufts University, 200 College Ave, Medford, USA - alexander.liss@ tufts.edu \\ ${ }^{d}$ Dept. of Community Health, Tufts University, 574 Boston Avenue, Medford, USA - karen.kosinski@ tufts.edu \\ ${ }^{\mathrm{e}}$ Neglected Tropical Disease Control Program, Ghana Health Service, Accra, Ghana - nanakwadwo.biritwum@ ghsmail.org \\ ${ }_{\mathrm{f}}^{\mathrm{f}}$ Friedman School of Nutrition Science and Policy, Tufts University, 150 Harrison Ave, Boston, USA - elena.naumova@tufts.edu
}

\section{Commission VIII, WG VIII/2}

KEY WORDS: Schistosomiasis Risk, Habitat Suitability Index, Domestic and Recreational Surface Water Use, Ghana

\begin{abstract}
:
Schistosoma haematobium transmission is influenced by environmental conditions that determine the suitability of the parasite and intermediate host snail habitats, as well as by socioeconomic conditions, access to water and sanitation infrastructure, and human behaviors. Remote sensing is a demonstrated valuable tool to characterize environmental conditions that support schistosomiasis transmission. Socioeconomic and behavioral conditions that propagate repeated domestic and recreational surface water contact are more difficult to quantify at large spatial scales. We present a mixed-methods approach that builds on the remotely sensed ecological variables by exploring water and sanitation related community characteristics as independent risk factors of schistosomiasis transmission.
\end{abstract}

\section{INTRODUCTION}

\subsection{Schistosomiasis transmission and control strategies}

Urogenital schistosomiasis is a water-related parasitic disease transmitted through skin contact with surface water containing Bulinus snails and human waste. It is known as a disease of poverty, with sub-Saharan Africa bearing approximately $90 \%$ of the disease burden (WHO, 2016). School-aged children are especially vulnerable to contracting the disease due to their role in fetching water for domestic uses and play habits such as swimming or fishing in infested water bodies (WHO, 2016). The most prominent schistosomiasis control strategy is periodic mass drug administration (MDA) with praziquantel, typically conducted through the school system. This approach limits severe health consequences of the disease later in life but does not prevent reinfection. In highly endemic areas where reinfection is common, this strategy is unlikely to achieve sustainable control (Secor, 2014). In recent literature, more comprehensive approaches including improvements in access to potable water and adequate sanitation, as well as snail elimination methods, have been recommended (Campbell et al., 2014; Grimes et al., 2015; King \& Bertsch, 2015; Secor, 2014; Steinmann et al., 2006).

Schistosomiasis is endemic to Ghana, with the majority of the country classified as having $10-50$ or $>50 \%$ prevalence of $S$. haematobium infection (or urogenital schistosomiasis), one of the two forms of the disease present in Africa (WHO, 2010). In 2008-2010, it was estimated that $30-35 \%$ of the population required preventive chemotherapy with praziquantel, with only $2-8 \%$ being treated each year (WHO, 2010). Although access to praziquantel has increased in recent years through Merck donation program facilitated by the World Health Organization (Merck, 2016), many people in Ghana and other parts of subSaharan Africa still lack access due to challenges with resource allocation.

\subsection{The need for predictive modelling}

Challenges in resource allocation for schistosomiasis control stem in part from the limitations of surveillance systems in lowincome countries to sufficiently detect the disease. Because it is largely a chronic condition for which people in rural settings are much less likely to seek treatment than for potentially fatal acute conditions like malaria, a large number of cases remains undetected and untreated (de Vlas et al. 2014). For example, in Ghana, it was estimated that the entire population, or $24,332,755$ individuals, required preventive chemotherapy in 2010 (WHO, 2010). However, only 24,996 cases (0.1\%) were reported into the Ghana Health Service electronic surveillance system for that year (GHS, 2016). Furthermore, health authorities of low-income countries lack the resources to carry out comprehensive prevalence screenings to track changes in endemicity over time and over small spatial scales. For example, the last comprehensive prevalence survey in Ghana

\footnotetext{
* Corresponding authors
} 
was carried out in the 1970s (Doumenge et al. 1987). Since then, a relatively small country-wide survey was conducted in 2008 in which school children in a total of 77 schools were screened, representing 62 of 138 districts (Soares-Magalhaes et al., 2011). Due to severe resource limitations in endemic countries, predictive modelling approaches are necessary to complement the limited surveillance data and prevalence surveys in order to develop targeted resource allocation plans and to inform strategies for sustainable schistosomiasis control.

\subsection{Summary of current modelling approaches}

Remote sensing is a valuable tool to characterize environmental conditions that support transmission of numerous infectious diseases, including schistosomiasis. Elevation, land surface temperature, precipitation, and vegetation indices derived from remote sensing data are among the most common environmental parameters associated with schistosomiasis risk (Walz et al., 2015a). The analytical approaches vary, as well as spatial and temporal resolutions of the data that they utilize. Recent reviews identified several limitations of the current modelling approaches including the following: 1) inability to spatially distinguish the locations of transmission vs. the locations from which prevalence estimates are derived (Walz et al., 2015a; Walz et al., 2015b); 2) insufficient spatial resolution to make the findings relevant to implementation agencies for targeted interventions; and 3) inability to incorporate social and behavioural conditions that promote surface water contact and reinfection (Simoonga et al., 2009).

\subsection{Mixed-methods approach}

To account for the identified limitations, we propose a mixed methods approach that builds on the current innovative ecological model that defines a habitat suitability index (HSI) of the intermediate snail host using remote sensing data (Walz et al., 2015c). We complement the HSI with community level attributes that affect human contact with potential transmission sites due to domestic and recreational surface water use. Using geospatial data from a field survey conducted in 74 rural communities, we characterize recreational exposure by defining a 'recreation potential' score of the water contact points. We also define improved water access and groundwater quality metrics for each town, which represent drivers of surface water use for domestic purposes. We evaluate domestic water use and recreational profiles of communities as independent risk factors for schistosomiasis transmission, in addition to the HSI. The novelty of the mixed-methods approach is a refined spatial scale (community or sub-community) and selection of parameters that reflect an on-the-ground understanding of schistosomiasis transmission attributes. If successful, this approach has the potential to identify community-specific intervention strategies to reduce the use of surface water for domestic and recreational purposes, and thereby reduce exposure to S. haematobium.

\section{METHODS}

\subsection{Study area and study design}

The study was conducted in the Eastern Region of Ghana, the third most populous of Ghana's ten administrative regions, with over half of the population (56.6\%) residing in rural areas (GSS, 2010). The Eastern Region lies in the deciduous forest agro-ecological zone, characterized by major and minor peak rainfall periods in June and October, respectively. The Harmattan, a persistent prevailing wind from the Sahara region, brings dry season from November to February. Major rivers of the Eastern Region include Pra, Birim, Ayensu, and Densu (Figure 1).

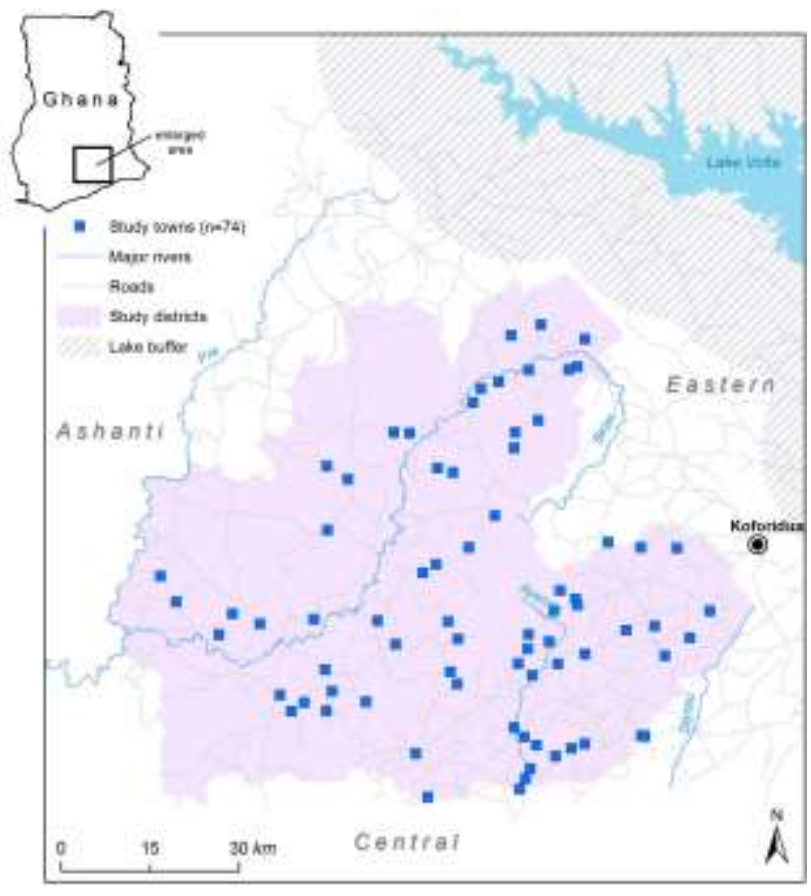

Figure 1: Map of study area

The Eastern Region is further sub-divided into 26 administrative districts. Ten adjacent districts were chosen as the study area because they were similar in their elevation profile, density of minor rivers and streams, land cover, and land use characteristics, and were outside of a $20-\mathrm{km}$ buffer of Lake Volta (Figure 1). The lake is a major schistosomiasis transmission site in Ghana; however, we were interested in studying schistosomiasis transmission along minor rivers and streams with domestic and recreational water contact.

To define the list of towns from which study towns were selected, the most recent settlement-level population data for the Eastern Region from the 2000 Census were obtained from the Ghana Statistical Service (GSS). Although a 2010 Census was conducted, the data have not yet been released at the town and village level. A data layer of settlement locations was also obtained from the Center for Remote Sensing and Geographic Information Services (CERSGIS). Census data were geocoded for all communities within the 10 districts in the target population range of $500-5,000$, according to the 2000 Census. In Ghana, localities with fewer than 5,000 inhabitants are termed rural (GSS, 2010). Communities with fewer than 500 residents were excluded. A total of 226 towns that met the population criteria were mapped.

Concurrently, Ghana Health Service (GHS) Disease Control offices were consulted for a list of known or suspected schistosomiasis-endemic towns, which were also mapped. From the 226 towns mapped in the study area, 74 (33\%) were purposively selected for the study. Purposive sampling consisted of selecting towns on the endemic list from the total list of mapped towns (41/74) and also sampling nearby towns with similar population characteristics that were not considered by GHS to be endemic (33/74). The sampling strategy resulted in a slight bias toward medium-sized towns in the 1,000-2,000 and 2,000 - 5,000 population categories (United Nations, 
2016). We subsequently projected the town populations to 2014 using a $2.1 \%$ annual population growth (GSS, 2010).

\subsection{Field data collection}

The first round of field data occurred between December 2013 and April 2014 by a team of two individuals (one native Twi speaker, one data recorder), along with a local guide, in each of the 74 towns. In each town, all publicly available and accessible water sources were documented, geocoded and mapped. Documented water sources included public standpipes (SPs) in piped water systems (PWSs), drilled boreholes (BHs), handdug wells (HDWs), and surface water access points (SWAPs). Brief surveys were also completed about BHs and SWAPs. Information was obtained verbally from community members in Twi and recorded in English.

$\mathrm{BH}$ attributes included reported water quality problems, that were were based on the responses from adult community members living near the BHs. Respondents were asked to comment on any water quality problems they had with the $\mathrm{BH}$ in an open-ended format. We opted for the open-ended format during this initial survey because no prior knowledge of water quality problems was available and to allow for all possible responses. For the SWAPs, relative depth, width and clarity of the water were recorded. Each access point was subsequently classified as 'perennial' if it contained water during the field visit, which occurred in the dry season.

During the first round of data collection, it was identified that the study area had problems with groundwater quality that were noted by the community members. Reported problems included oil separation, unfavorable scent, plantain turning black/purple when cooked with borehole water, salty taste, particulate matter, and soap not lathering when doing laundry. A follow-up study systematically collected self-reported water quality information as well as corresponding measured water quality data during dry and rainy seasons from 94 boreholes across the study area. The water samples were analyzed for 26 common physicochemical water quality parameters. In this study, it was found that oil separation, unfavourable scent and plantain staining were concurrently reported problems associated with high iron content. Salty taste and soap not lathering, as expected, were associated with total dissolved solids and total hardness concentrations of the water, respectively.

Finally, a self-reported prevalence study was conducted from September to November 2015. The study consisted of two surveys in each visited public primary school and included three self-reported variables (fetching of or swimming in surface water and having seen hematuria in urine in the past two weeks) collected from all children in grades 3 through 6 in 73 primary schools; two of the 74 study towns shared a school. A follow-up survey was also conducted in a subset of 30 of the 73 initial schools and involved 30 children in grades 3 and 4 only. During the second survey, the same three self-reported variables were collected and matched with a urine sample using a random ID. The urine samples were tested for presence of hematuria and $S$. haematobium eggs (Kosinski et al. 2011). The results of this second survey are not used in the present analysis.

\subsection{Definition of analysis variables}

\subsubsection{Habitat suitability index}

Environmental conditions relevant for disease transmission such as stagnant or slow moving water bodies, riparian vegetation, and water surface temperatures have been derived using remote sensing data from the Landsat 8 and Sentinel 2A satellites, as well as the $30 \mathrm{~m}$ Advanced Spaceborne Thermal Emission and Reflection Radiometer Global Digital Elevation Model (ASTER GDEM) and integrated into an HIS (Walz et al., 2015c).

\subsubsection{Improved water coverage}

The GPS coordinates of all improved public water sources (i.e. SPs, BHs, and HDWs) were combined into a single data set. Improved water coverage is defined as the percent of a population with access to an improved water source, according to the UNICEF/WHO Joint Monitoring Programme (JMP) definition. For our purposes, we considered unimproved handdug wells in our definition of improved (excluded in the JMP definition) because they are not possible $S$. haematobium transmission sites. In order to calculate percent coverage, two population data sources were used. As an additional objective of the study, we compared the estimates of water coverage using multiple sources of population data: 1) town polygons exported from Open Street Map normalized by the Census population, assuming uniform population density and 2) WorldPop population density raster grid data with $100 \mathrm{~m}^{2}$ spatial resolution (Linard et al., 2012). Percent improved water coverage was calculated for 100, 200, 300, 400 and 500 meter buffer distances from the point sources. Separate analyses were conducted using all improved water sources and only functional improved water sources. These metrics were compared against more precise estimates provided using roof reflectance values derived from a Google Earth base image in ENVI software (version 4.8) as proxies of populated $1 \mathrm{~m}^{2}$ pixels normalized by the Census population, assuming uniform population density, and using actual mapped households as a method of calibration.

\subsubsection{Groundwater quality}

Three water quality parameters were derived based on results of the prior water quality study. These parameters were total iron $(\mathrm{mg} / \mathrm{L})$, total dissolved solids $(\mathrm{mg} / \mathrm{L})$, and total hardness $(\mathrm{mg} / \mathrm{L}$ as $\mathrm{CaCO}_{3}$ ). Surfaces of each constituent's concentrations over the study area were derived using spatial interpolation in ArcGIS (version 10.2.2). Subsequently, average concentration for each study town was extracted using the Open Street Map town polygons and zonal statistics tools in ArcGIS.

\subsubsection{Recreation score}

A recreation score was derived from the following parameters available from field survey: water depth, clarity, width of the stream and whether or not it was present in the dry season. Deeper, wider, and clearer streams that were present in the dry season were categorized as higher risk. A cumulative score for each town that accounted for the total number of SWAPs was then calculated as a sum of individual SWAP scores.

\subsubsection{Outcome measures}

The percentage of children who self-reported fetching surface water, swimming in surface water, and seeing blood in their urine within two weeks of the survey were considered as intermediate outcomes because they were available for all 73 study towns (one town was dropped from the analysis because it did not have a school). In the full analysis, parasitological 
prevalence measures will be considered as the outcome of interest.

\section{RESULTS}

\subsection{Improved water coverage}

The case study showed that for a relatively small town of approximately 2,000 people, all measures of population density yielded similar estimates of water coverage (Figure 2). The town polygons slightly under-approximated and WorldPop slightly over-approximated water coverage. The roof reflectance values yielded nearly identical results as mapped households. For a larger town however, with approximately 5,000 people, the WorlPop estimate provided a gross underestimate of water coverage as compared to the other methods. However, mapped households were not available for large towns for a complete comparison (Figure 3).

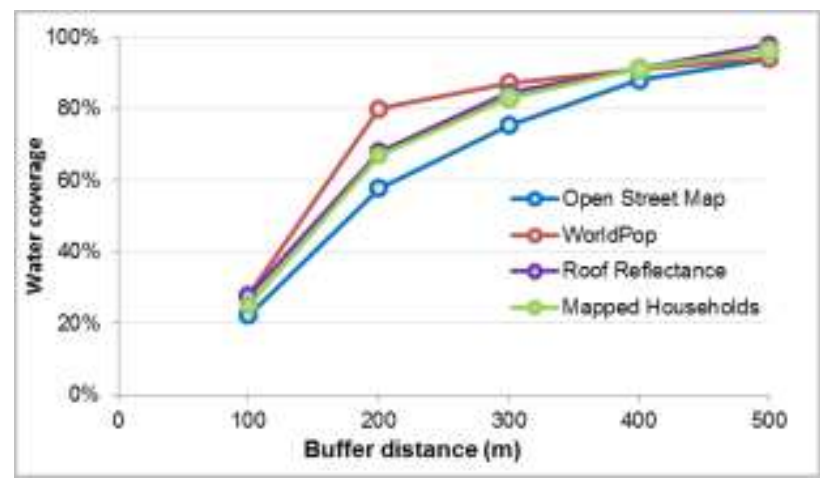

Figure 2: Comparison of water coverage calculated using four population data sources for a small town $(\sim 2,000$ people $)$

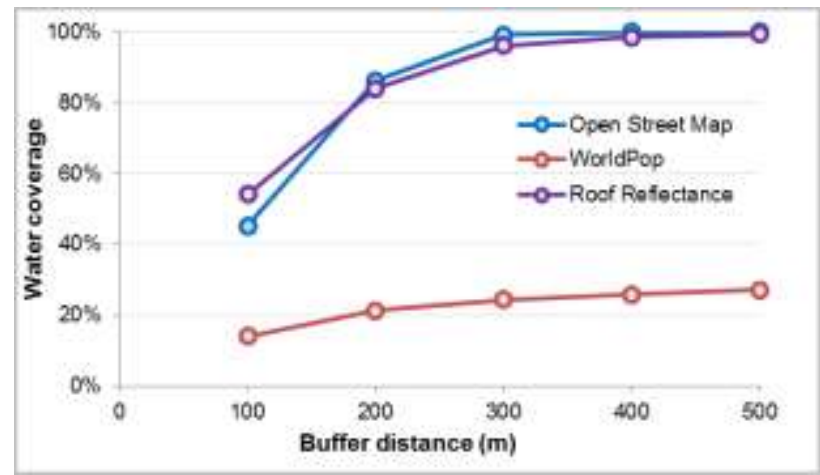

Figure 3: Comparison of water coverage calculated using three population data sources for a medium town $(\sim 5,000$ people $)$

Overall, water coverage that was estimated using the two population methods trended similarly, with WorldPop providing on average a $10 \%$ lower estimate for 200 meter buffer distance and 20\% lower estimate for 500 meter buffer distance as compared to the Open Street Map polygons (Figure 4). Between 60 and $80 \%$ of the study population overall lives within 300 meters of an improved water source. If only functional water sources are considered, this estimate drops down to $58-70 \%$. Similarly, Between 80 and $95 \%$ of the study population overall live within 500 meters of an improved water source. If only functional water sources are considered, this estimate drops down to $75-90 \%$ (Figure 4).

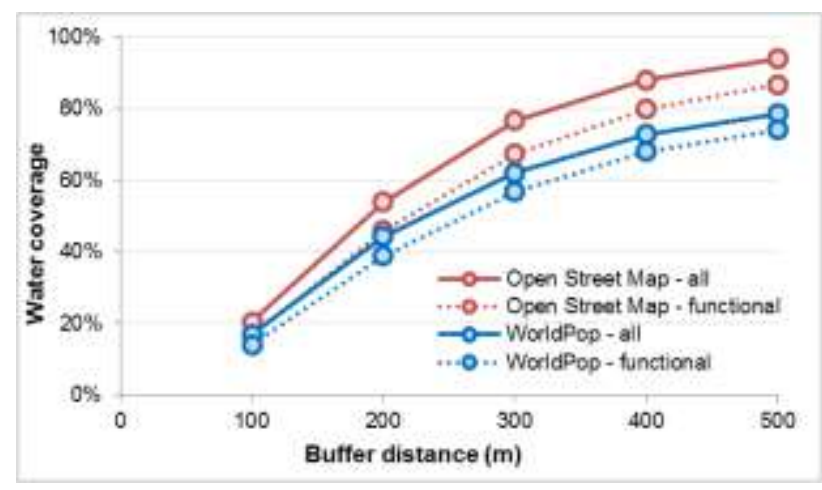

Figure 4: Comparison of water coverage calculated using all and only functional water sources and two population data sources for all towns

When improved water coverage was used as a single predictor of self-reported fetching of surface water in a linear model (both variables were log-transformed to achieve a normal distribution), water coverage using both population estimates and using all and only functional water sources was negatively associated with the percentage of school children who reported fetching surface water. This association is in the direction expected, i.e. higher water coverage results in lower rates of surface water use; however, it was not statistically significant.

\subsection{Groundwater quality}

Predicted surfaces of iron, TDS and hardness concentrations are presented in Figure 5. Predicted iron concentrations ranged between 0.05 and $1.15 \mathrm{mg} / \mathrm{L}$ (mean $=0.35, \mathrm{SD}=0.27$ ). Predicted TDS concentrations ranged between 77 and $626 \mathrm{mg} / \mathrm{L}$ $($ mean $=234, \mathrm{SD}=150)$. Predicted hardness concentrations ranged between 46 and $313 \mathrm{mg} / \mathrm{L}($ mean $=111, \mathrm{SD}=62)$. All of the groundwater quality problems exhibit visual clustering with iron concentrations occurring in the northern part of the study area and TDS and hardness occurring in the south-eastern corner of the study area.

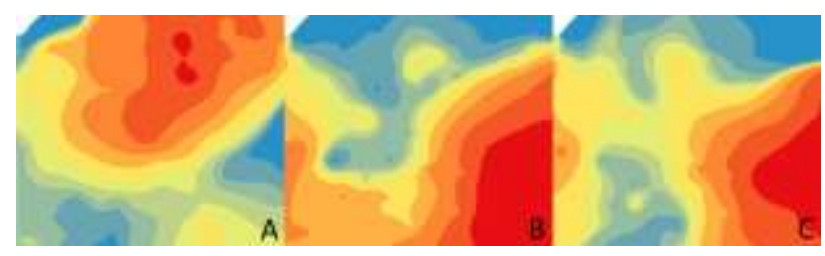

Figure 5: Interpolated iron (A), total dissolved solids (B) and total hardness $(\mathrm{C})$ concentrations in groundwater samples.

When each water quality parameter was used as a single predictor of self-reported fetching of surface water in a linear model (both variables were log-transformed to achieve a normal distribution), the association between self-reported fetching and iron concentration was not statistically significant. TDS and hardness concentrations, however, were positively associated with the percentage of school children that reported fetching surface water. This association is in the direction expected, i.e. higher concentrations of undesirable constituents in improved water sources results in higher rates of surface water use. A one percent increase in TDS concentration was associated with $0.17 \%$ increase in the percentage of self-reported fetching of surface water $(0<0.01)$. A one percent increase in hardness concentration was associated with $0.19 \%$ increase in the percentage of self-reported fetching of surface water $(0<0.01)$. 


\subsection{Recreation score}

The recreation score values per surface water access point ranged between 2 and $10($ mean $=7.5, \mathrm{SD}=1.5)$. On a town level, the total recreation score ranged between 0 (when a town did not have any surface water access points) and 59 (mean = $20.5, \mathrm{SD}=14.5)$. When recreation score was used as a single predictor of self-reported swimming in surface water in a linear model (both variables were log-transformed to achieve a normal distribution), recreation score was positively associated with the percentage of school children who reported swimming in surface water. This association is in the direction expected; one percent increase in recreation score was associated with $0.14 \%$ increase in the percentage of self-reported swimming in surface water $(0<0.01)$.

\subsection{Future directions}

The next steps for this analysis are to calculate the HSI for the study area and combine it with the three community predictors of interest in a final model where parasitological UGS prevalence is the outcome variable. The HSI will be field validated. The model will be conducted with and without accounting for potential spatial autocorrelation in the data. More work is also necessary in defining the recreation score as well as selecting the most appropriate methods of calculating improved water coverage.

The methods we propose here have implications for sustainable schistosomiasis control. The methods require field data collection, but the surveys utilized were brief, not resourceintensive, and can be carried out for a larger geographic area. Furthermore, the risk factors we consider have practical applications in that they allow for categorization of towns according to potential intervention strategies. For example, towns with low water coverage, high levels of undesirable groundwater constituents, and a high recreation score should be prioritized for routine MDA as well as primary prevention measures.

\section{ACKNOWLEDGEMENTS}

This study was funded in part by the National Institutes of Health 1 R34 AI097083-01A1, Tufts Institute for Innovation, Natalie V. Zucker, and Charlton research grants. We would like to thank members of Ghana Health Service, Ghana Education Service, and Community Water and Sanitation Agency for their ongoing support of our research activities in the Eastern Region. We would also like to thank Prof. Kwabena M. Bosompem and Dickson Osabutey of Noguchi Memorial Institute for Medical Research, community liaison Michael N. Adjei, as well as students Ariel Branz, Madeline Wrable, Michelle Sodipo and Paige Roberts of Tufts University, and Bernard Osei and Emmanuel Asare of University College of Agriculture and Environmental Studies for their assistance in the field. We would also like to thank community members and school children of the study towns for participating in our studies.

\section{REFERENCES}

Campbell SJ, Savage GB, Gray DJ, Atkinson JAM, Soares Magalhaes RJ, Nery SV, McCarthy JS, Vellenman, Y, Wicken JH, Traub RJ, Williams GM, Andrews RM, Clements ACA. Water, Sanitation, and Hygiene (WASH): A critical component for sustainable soil-transmitted helminth and schistosomiasis control. PLoS Neglected Tropical Diseases 2014, 8: e2651.

De Vlas SJ, Danso-Appiah A, van der Werf MJ, Bosompem KM, Habbema JDF: Quantitative evaluation of integrated schistosomiasis control: the example of passive case finding in Ghana. Trop Med Int Health 2004, 9(6):A16-A21.

Doumenge JP and WHOPD Programme, 1987. Atlas of the global distribution of schistosomiasis, Presses Universitaires de Bordeaux Bordeaux, France.

Ghana Statistical Service. 2010 Population and housing Census: National analytical report. 2013 ; http://www.statsghana.gov.gh/pop_stats.html.

Grimes JET, Croll D, Harrison WE, Utzinger J, Freeman MC, Templeton MR. The role of water, sanitation and hygiene in reducing schistosomiasis: a review. Parasites \& Vectors 2015 , 8: 156.

King CH \& Bertsch D. Historical Perspective: Snail Control to Prevent Schistosomiasis. PLoS Neglected Tropical Diseases 2015, 9(4): e0003657.

Kosinski KC, Bosompem KM, Stadecker MJ, Wagner AD, Plummer J, Durant JL, Gute DM: Diagnostic accuracy of urine filtration and dipstick tests for Schistosoma haematobium infection in a lightly-infected population of Ghanaian schoolchildren. Acta Trop 2011, 118(2):123-127.

Linard C, Gilbert M, Snow RW, Noor AM, Tatem AJ. Population distribution, settlement patterns and accessibility across Africa in 2010. PLoS One 2012, 7(2): e31743.

Merck KGaA. Fighting Schistosomiasis (accessed 04/2016). http://www.emdgroup.com/emd/responsibility/society/schistoso miasis/schistosomiasis.html.

Simoonga C, Utzinger J, Brooker S, Vounatsou, P, Appleton CC, Stensgaard AS, Olsen A, Kristensen TK. Remote sensing, geographical information system and spatial analysis for schistosomiasis epidemiology and ecology in Africa. Parasitology 2009, 136(13): 1683-1893.

Soares Magalhaes RJ, Biritwum NK, Gyapong JO, Brooker S, Zhang Y, Blair L, Fenwick A, Clements AC. Mapping helminth co-infection and co-intensity: geostatistical prediction in Ghana. PLoS Neglected Tropical Diseases 2011, 5: e1200.

Steinmann P, Keiser J, Bos R, Taner M, Utzinger J. Schistosomiasis and water resources development: systematic review, meta-analysis, and estimates of people at risk. The Lancet Infectious Diseases 2006, 6: 411-425.

United Nations. Population density and urbanization (accessed January 2016)

http://unstats.un.org/unsd/Demographic/sconcerns/densurb/dens urbmethods.htm.

Walz Y, Wegmann M, Dech S, Raso G, Utzinger J. Risk profiling of schistosomiasis using remote sensing: approaches, challenges and outlook. Parasites \& Vectors 2015, 8: 163 (a).

Walz Y, Wegmann M, Leutner B, Dech S, Vountsou, P, N'Goran EK, Raso G, Utzinger J. Use of an ecologically 
relevant modelling approach to improve remote sensing-based schistosomiasis risk profiling. Geospatial Health 2015, 10: 398 (b).

Walz Y, Wegmann M, Dech S, Vounatsou P, Poda JN, N'Goran EK, Utzinger J, Raso G. Modeling and validation of environmental suitability for schistosomiasis transmission using remote sensing. PLoS Neglected Tropical Diseases 2015, 9(11): $\mathrm{e} 0004217$ (c)

WHO. Schistosomiasis Fact Sheet (accessed 04/2016). http://www.who.int/mediacentre/factsheets/fs115/en/.

WHO. Ghana Country Profile 2010 (accessed 04/2016). http://www.who.int/neglected_diseases/preventive_chemothera py/databank/CP_Ghana.pdf?ua=1. 